\title{
Intérêt des analyses toxicologiques lors d'une recherche des causes de décès (résultats de 358 analyses)
}

\section{Interest of toxicological analysis in the research of case of death (results of 358 analysis)}

Charlotte DUVERNEUIL ${ }^{(1)}$, Isabelle ETTING( ${ }^{(1)}$, Bertille MATHIEU ${ }^{(1)}$,

\section{François PARAIRE(2), Geoffroy LORIN DE LA GRANDMAISON ${ }^{(2)}$, Caroline RAIMBAULT ${ }^{(2)}$, Michel DURIGON ${ }^{(2)}$, Philippe de MAZANCOURT ${ }^{(1)}$, Jean-Claude ALVAREZ ${ }^{(1) *}$}

(1) Laboratoire de Pharmacologie - Toxicologie (2) Service de Médecine Légale, Centre Hospitalier Universitaire Raymond Poincaré, AP-HP, 104, Boulevard R. Poincaré- 92380 GARCHES

*Auteur à qui adresser la correspondance : Jean-Claude ALVAREZ, Laboratoire de Pharmacologie-Toxicologie, Centre Hospitalier Universitaire Raymond Poincaré, 104, Boulevard R. Poincaré - 92380 GARCHES Tél : 0147107938 - Fax : 0147107923

(Reçu le 12 avril 2005 ; accepté après modifications le 12 août 2005)

\section{RESUMÉ}

Trois cent cinquante-huit prélèvements post-mortem, représentant 30 mois d'expertises toxicologiques, ont été analysés afin de déterminer l'intérêt des résultats dans le cadre des recherches de causes de décès. Nous avons pour cela comparé les résultats d'analyses aux conclusions établies lors de l'autopsie lorsque celle-ci était pratiquée $(n=292)$. Dans 66 cas, l'autopsie n'a pas été effectuée, les prélèvements ayant été réalisés lors de "levée de corps ». La présence d'au moins un xénobiotique est retrouvée dans 272 cas (76\%) sur les 358 décès. Sur les 80 décès pour lesquels la conclusion de l'autopsie était une origine toxique probable, 68 (85\%) ont été confirmés par l'analyse, 12 cas (15\%) ne révélant pas de toxiques. Sur les 181 décès pour lesquels la cause était

\section{SUMMARY}

358 post-mortem samples over a 30 months follow-up periode have been analysed in order to establish the interest of toxicological analysis in the research of cause of death. We have also compared the analysis results with the autopsy conclusions when available ( $n=292)$. In 66 cases, the autopsy has not been carried out, the samples being collected on the site of death. The presence of at least one xenobiotic was found in 272/358 cases ( $76 \%$ ). Concerning the 80 deaths for which the autopsy conclusion could be attributed to toxic origin, 68 (85\%) have been confirmed by the analysis, 12 cases (15\%) did not evidence any toxic. Among the 181 deaths where the cause appeared to be obvious during autopsy (drowning, homicide or hanging), 112 (61,8\%) 
évidente à l'autopsie et a priori d'origine non toxique, par exemple par noyade, homicide ou pendaison, $112(61,8 \%)$ ont révélé l'absence de tout toxique et 69 (34,2\%) montraient la présence de toxiques pouvant avoir joué un rôle dans les circonstances du décès. Sept de ces décès pouvaient même être directement expliqués par la seule présence des toxiques. Enfun, sur les 31 décès d'origine naturelle probable, 27 (87\%) ont montré l'absence de tout toxique et 4 (13\%) ont révélé un décès d'origine toxique. Les médicaments sont les substances les plus souvent rencontrées (168 décès, soit $61,7 \%)$, essentiellement représentés par les benzodiazépines (27\%) et les antidépresseurs $(16,2 \%)$. L'alcool est retrouvé dans 158 cas (58\%). Les stupéflants sont retrouvés dans 62 cas $(22,7 \%)$, le cannabis étant le plus souvent rencontré $(n=50)$, devant la morphine $(n=21)$, dont $15 \mathrm{impu-}$ tables à une prise d'héroïne (présence de 6-MAM et codéine).

\section{MOTS-CLÉS}

Analyses toxicologiques, recherche des causes de décès, xénobiotiques, statistiques.

\section{Introduction}

Les causes de décès sont déterminées dès l'étape de l'autopsie dans de nombreux cas, notamment dans la plupart des morts violentes, traumatiques ou instrumentales (blessures par arme blanche ou par arme à feu, pendaisons, strangulations, accidents de la circulation...). Toutefois, pour certaines autopsies, persiste parfois le doute d'une association avec des toxiques, par exemple lors d'une noyade. En effet, il est possible que l'absorption d'une ou plusieurs substances psychoactives puisse entraîner certains troubles de la vigilance, voire de la conscience, pouvant avoir pour conséquence la survenue du décès. Par ailleurs, certaines autopsies ne permettent pas d'établir la cause du décès (absence de lésions de violence et de lésions organiques). Une expertise toxicologique est, dans ces cas, souvent demandée afin d'expliquer le décès. Même si parfois cette expertise ne permet pas davantage de conclure à la cause de décès (absence de xénobiotiques ou quantité trop infime de prélèvements à disposition), elle apporte, la plupart du temps, des renseignements très importants aboutissant soit à l'explication du décès (comme pour une noyade), soit à l'établissement de la cause du décès (mort toxique, suicide médicamenteux). Ainsi, dans une précédente étude (1), les expertises toxicologiques avaient permis d'établir la cause de la mort (décès imputables à la buprénorphine) alors que les autopsies avaient mis en évidence uniquement des signes d'asphyxie (aucune autre cause de décès n'ayant pu être retenue par des médecins légistes expérimentés). Ces expertises toxicologiques sont parfois également demandées longtemps après le décès (lors d'exhumation des corps) et permettent ainsi de déceler un empoi- revealed the absence of toxic. The other 69 deaths (34,2\%) showed the presence of toxics, that could be involved in the cause of death. Seven of these deaths could be directly explained by the presence of toxics. Finally, 31 deaths were listed with a natural origin. Among these 31 deaths, 27 (87\%) revealed the absence of toxic and 4 (13\%) showed a toxic death. The pharmaceuticals were the substances the most frequently detected (168 deaths, 61,7\%), essentially benzodiazepines (27\%) and antidepressants $(16,2 \%)$. Alcohol was found in 158 cases (58\%). Drugs of abuse were observed in 62 cases (22,7\%), essentially cannabis ( $n=50$ ), then morphine ( $n=21$ including 15 with a intake of heroin characterised by the presence of 6-MAM and codeine).

\section{KEY-WORDS}

Toxicological analysis, determination of cause of death, xenobiotics, statistics.

sonnement alors que les circonstances de la mort étaient restées inaperçues au départ (2). L'importance de ces analyses toxicologiques est par ailleurs reconnue dans d'autres pays puisqu'en Finlande, par exemple, toute mort susceptible d'être toxique est soumise systématiquement à une expertise toxicologique (3).

Le but de cette étude est de démontrer l'importance de l'analyse toxicologique consécutive à une autopsie, et d'établir les relations entre les résultats toxicologiques et les conclusions d'autopsies. Par ailleurs, cette étude permet également de recenser les molécules les plus fréquemment rencontrées dans des affaires d'autolyse, d'empoisonnement ou encore d'accidents, un tel bilan n'ayant pas été effectué en France depuis l'étude lyonnaise de Pascal et coll en 1996 (4).

\section{Matériel et méthodes}

\section{Population étudiée}

Les prélèvements analysés proviennent d'autopsies réalisées pour la plupart dans le service d'AnatomiePathologique du CHU R. Poincaré de Garches entre juin 2001 et décembre 2003. Dans ce cas, les conclusions de l'autopsie ont été systématiquement collectées. Les autres prélèvements proviennent de levées de corps pour lesquels une autopsie n'a pas été réalisée. Les résultats toxicologiques des levées de corps n'ont pas été retenus pour l'établissement des relations entre les résultats toxicologiques et les conclusions d'autopsies (étude de 272 dossiers).

Au total, les prélèvements de 358 autopsies ou levées de corps ont été analysés, demandés par le parquet et 
correspondant le plus souvent à des décès d'origine a priori non naturelle. Pour les dossiers les plus complets, nous avions à notre disposition du sang, des urines, du contenu gastrique, de la bile (prélèvements analysés en systématique) et d'autres organes (non analysés). Pour certains dossiers, nous n'avions que du sang ou que des organes (muscle et foie en général).

\section{Recherche et/ou dosage de stupéfiants}

\section{Dépistage urinaire}

Lorsqu'un prélèvement urinaire est disponible, un dépistage rapide par technique immunochimique sur automate AXSYM (Abbott ${ }^{\circledR}$ ) est réalisé pour les opiacés (anticorps dirigé contre la morphine), la cocaïne (anticorps dirigé contre la benzoylecgonine), les amphétamines (anticorps dirigé contre l'amphétamine et la métamphétamine), le cannabis (anticorps dirigé contre l'acide 11-nor-tétrahydrocannabinol-carboxylique) et la méthadone (anticorps dirigé contre la méthadone). Une recherche de buprénorphine est réalisée par technique immunochimique Elisa en microplaque (Microgenics $®$ ) utilisant un anticorps dirigé contre la buprénorphine.

\section{Confirmation et dosage des stupéfiants dans le sang et l'urine}

Tout résultat positif lors du dépistage est confirmé par la chromatographie en phase gazeuse couplée à la spectrométrie de masse (CPG/SM) ou chromatographie liquide couplée à une spectrométrie de masse en tandem (CL/SM/SM) pour la buprénorphine.

\section{Recherche et/ou dosage de médicaments}

Recherche non spécifique par chromatographie en phase gazeuse couplée à la spectrométrie de masse (CPG/SM).

Après extraction acide et basique à partir des milieux biologiques en utilisant une association de quatre solvants (éther/dichlorométhane/hexane/alcool isoamylique, $250 \mathrm{ml} / 150 \mathrm{ml} / 100 \mathrm{ml} / 2,5 \mathrm{ml}$ ), les extraits méthanoliques obtenus avant et après acétylation sont injectés dans le chromatographe. L'identification des composés est obtenue par comparaison des temps de rétention chromatographique et des spectres de masse des molécules éventuellement trouvées avec celles présentes dans des bibliothèques de référence (NIST et PMW Maurer version1.7).

Recherche non spécifique par chromatographie en phase liquide couplée à la spectrophotométrie UV utilisant un détecteur à barrette de diodes (CLHP/UV-BD)

Les $50 \mu \mathrm{l}$ de l'extrait méthanolique préalablement préparé pour la CPG/SM sont évaporés et repris par $100 \mu \mathrm{l}$ d'un mélange tampon phosphate $20 \mathrm{mM}$ /acétonitrile (70/30). $50 \mu \mathrm{l}$ sont injectés dans le chromatographe.

L'identification des composés est obtenue par comparaison des temps de rétention chromatographique et des spectres UV des produits éventuellement trouvés, avec ceux stockés dans une bibliothèque réalisée au laborátoire et contenant plus de 700 spectres de médicaments, toxiques et stupéfiants constamment réactualisée.

\section{Méthodes spécifiques d'une classe thérapeutique}

La recherche toxicologique non spécifique précédemment décrite ne permettant de détecter que des concentrations relativement élevées de médicaments, des méthodes complémentaires spécifiques de certaines classes sont réalisées. Ces techniques spécifiques permettent également le dosage des molécules mises en évidence lors de la recherche non spécifique.

Les antidépresseurs tricycliques sont dosés en CPG/SM, les antidépresseurs non-tricycliques en CLHP/BD (5). Les neuroleptiques sont dosés soit en $\mathrm{CPG} / \mathrm{SM}$, soit en CLHP/BD, les benzodiazépines en CL/SM/SM, les béta-bloquants en CLHP/BD (6), les barbituriques en CPG/SM. Le dépistage des digitaliques (digoxine-digitaline) est réalisé par technique immunochimique sur automate AXSYM (Abbott@). La confirmation est réalisée en CL/SM/SM. Le dosage de lithium est réalisé à l'aide d'un photomètre de flamme et celui de la carboxyhémoglobine est effectué par l'intermédiaire d'un hémoxymètre $\mathrm{ABL} 700$ (Radiometer). Le dosage de l'alcool est réalisé par chromatographie en phase gazeuse couplée à une détection par ionisation de flamme. Le propanol-1 est utilisé comme étalon interne.

Cette liste de techniques est non exhaustive puisque d'autres recherches plus spécifiques peuvent être réalisées en fonction du contexte (dosage de paracétamol, de dextropropoxyphène, de cyanures, recherche de substances volatiles...).

\section{Résultats et discussion}

\section{Substances mises en évidence}

La présence d'un xénobiotique est retrouvée dans 272 des 358 autopsies ou levées de corps étudiées (76\%). Nous avons choisi de regrouper les substances retrouvées en 6 catégories (figure 1) à savoir les médicaments, l'alcool, les stupéfiants, les médicaments de substitution, le monoxyde de carbone ( $\mathrm{CO}$ ) et une dernière catégorie nommée « autres ».

\section{Les médicaments (figure 2)}

Les médicaments sont les substances les plus fréquemment rencontrées puisque dans 168 cas $(61,7 \%)$ des 


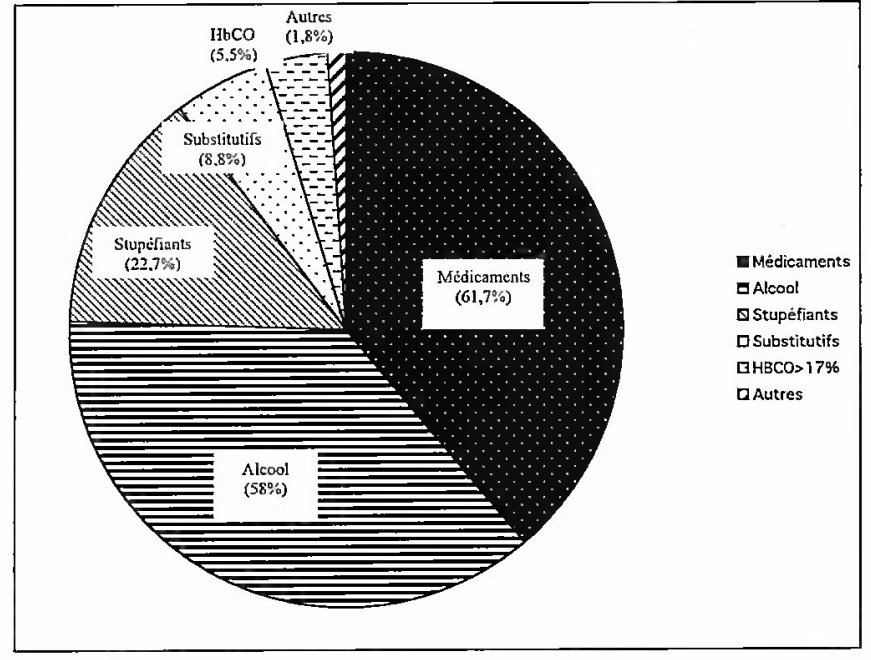

Figure 1 : Types de substances retrouvées.

dossiers analysés, au moins un médicament est retrouvé. Parmi ces médicaments, les plus fréquents sont les benzodiazépines (en particulier le diazépam, le nordiazépam, l'oxazépam et le bromazépam) retrouvées dans 97 cas $(27 \%)$. Le succès des benzodiazépines, comme le soulignent Tracqui et Ludes (7), s'explique par une efficacité reconnue dans plusieurs domaines donc une prescription très importante, une réputation de grande sécurité et un faible coût. Viennent ensuite les antidépresseurs retrouvés dans 58 cas $(16,2 \%)$ avec essentiellement de la clomipramine, de la fluoxétine et du citalopram. Il est admis que le risque suicidaire chez les patients déprimés traités persiste au moins durant les 15 premiers jours de traitement, la levée de l'inhibition psychomotrice pouvant précéder l'action antidépressive proprement dite du médicament, ceci pouvant expliquer la forte proportion d'antidépresseurs retrouvés lors de suicide. Les neuroleptiques, quant à eux, sont retrouvés dans 41 cas $(11,5 \%)$, surtout la cyamémazine, l'alimémazine, et l'acéprométazine.

Neuf dossiers comportent des béta-bloquants $(2,5 \%)$. Le paracétamol $(n=17)$, le méprobamate $(n=15)$, l'acide valproïque $(n=13)$, le dextropropoxyphène $(n=11)$, le tramadol $(n=8)$ sont les autres médicaments les plus souvent retrouvés. L'atracurium (à concentration thérapeutique, probablement administré en ante-mortem lors de la prise en charge médicale) a été retrouvé quatre fois. Un seul cas de barbiturique a été retrouvé, en l'occurrence du phénobarbital.

Ces résultats diffèrent de la précédente étude réalisée en 1996 par Pascal et coll. (4), le méprobamate arrivant, dans cette étude, en seconde position des médicaments les plus fréquemment rencontrés. Cette différence de résultats montre, comme le mentionnaient les auteurs, l'importance d'établir régulièrement un bilan des toxiques rencontrés au cours des expertises toxicologiques.

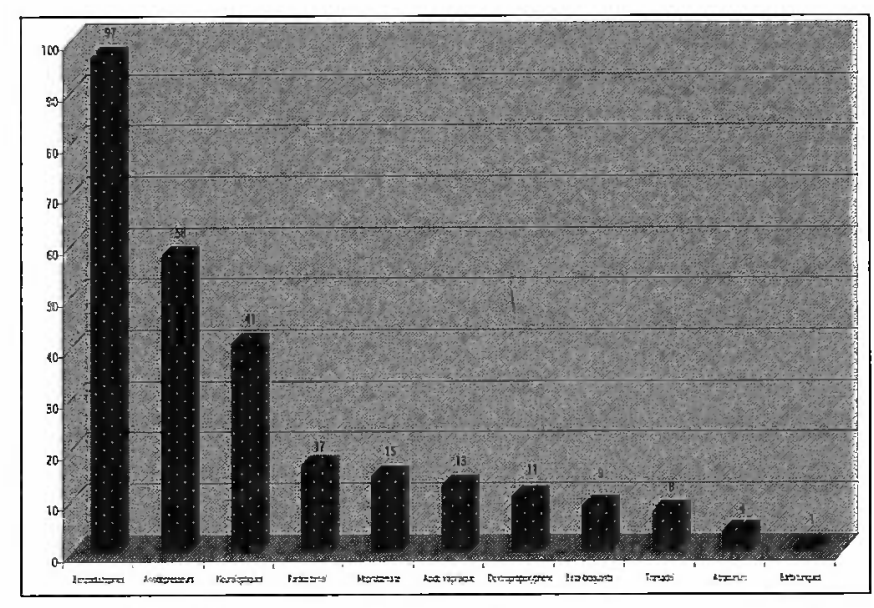

Figure 2 : Médicaments les plus fiéquemment rencontrés.

\section{L'alcool}

Parmi les 158 cas (58\%) dans lesquels de l'alcool est retrouvé, l'alcoolémie est inférieure à $1 \mathrm{~g} / \mathrm{l}$ dans 75 cas $(47,5 \%)$, entre 1 et $2 \mathrm{~g} / \mathrm{l}$ dans 47 cas $(29,7 \%)$ et supérieure à $2 \mathrm{~g} / \mathrm{l}$ dans 36 cas $(22,7 \%)$.

\section{Les stupéfiants (figure 3)}

Dans 62 cas parmi les 358 analysés, au moins un stupéfiant a été mis en évidence $(22,7 \%)$. Le cannabis est le plus souvent rencontré $(n=50)$, suivi de la morphine $(\mathrm{n}=21)$, dont 15 imputables à une prise d'hérö̈ne (présence de morphine, de 6-monoacétylmorphine et de codéine). Viennent ensuite la cocaïne ( $n=9)$, les amphétamines ( $\mathrm{n}=5$ ), la pholcodine (un cas) et l'éphédrine (un cas).

L'étude réalisée en 1996 par Pascal et coll. (4) mettait en évidence une prépondérance écrasante des opiacés et notamment d'héroïne parmi les stupéfiants retrouvés, alors que le cannabis est nettement prépondérant dans

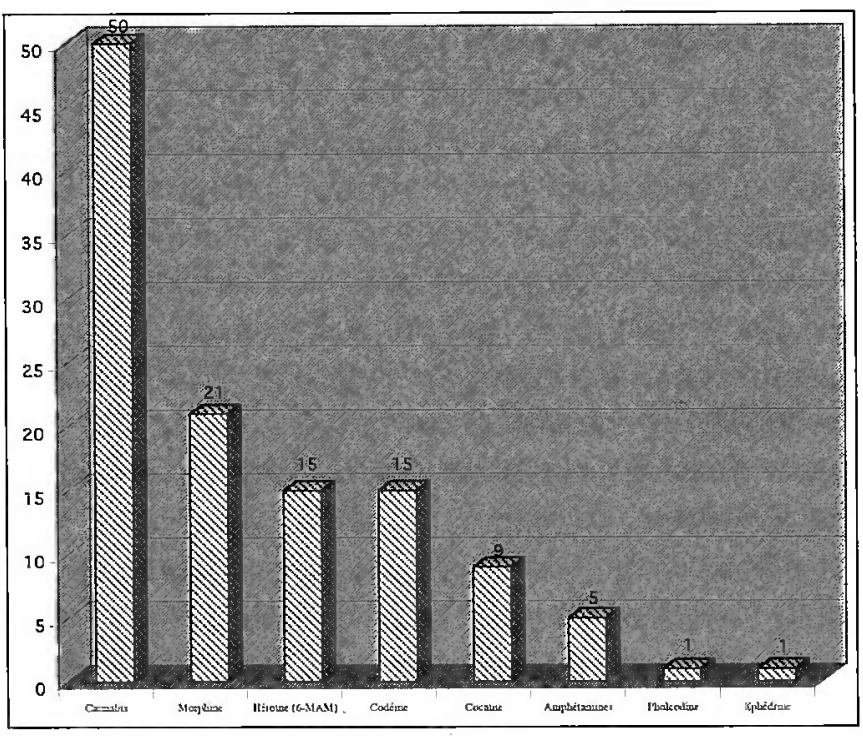

Figure 3 : Stupéfiants les plus fréquemment rencontrés. 
notre étude. Ce phénomène de recrudescence du cannabis est inquiétant, car même si « on ne meurt pas du cannabis... il tue ! (8) », dans la mesure où sa consommation entraîne bien souvent des troubles du comportement pouvant être la cause d'un décès. La plus faible proportion de décès à l'héroïne retrouvée dans notre étude est probablement le reflet de la forte diminution de consommation de cette substance en France.

\section{Les traitements de substitution (substitutifs) aux opia- cés}

Les traitements de substitution aux opiacés (méthadone $\mathrm{n}=11$, buprénorphine $\mathrm{n}=10$ ou les deux $\mathrm{n}=2$ ) sont retrouvés dans 24 cas $(8,8 \%)$. Dans 5 cas, les concentrations de méthadone étaient thérapeutiques (comprises entre 5 et $158 \mathrm{ng} / \mathrm{ml}$ ), et toxiques dans 8 cas ( 355 à $2330 \mathrm{ng} / \mathrm{ml}$ ). Pour la buprénorphine, 7 cas présentaient des concentrations thérapeutiques (entre 0,4 et 5 $\mathrm{ng} / \mathrm{ml})$ et 5 des concentrations toxiques $(7,4$ à 20 $\mathrm{ng} / \mathrm{ml}$ ). La présence relativement importante de méthadone peut s'expliquer par sa forte utilisation dans le traitement substitutif des pharmacodépendances majeures aux opiacés. Du fait de sa disponibilité, son usage détourné peut être la cause de décès. La prévalence similaire de buprénorphine peut être expliquée d'une part par son utilisation croissante comme analgésique (elle est de plus en plus souvent préférée à la morphine) dans le traitement des douleurs post-opératoires aiguës ou chroniques (cancéreuses), et surtout par son utilisation dans le traitement des dépendances aux opiacés qui s'est généralisée ces dernières années.

\section{Le monoxyde de carbone}

Une carboxyhémoglobine supérieure à $15 \%$ est retrouvée dans 15 cas $(5,5 \%)$ (un taux d'HbCO supérieur à $15 \%$ étant considéré cliniquement comme le reflet d'une intoxication aiguë), avec des taux compris entre $17 \%$ et $77 \%$.

\section{Autres}

De la strychnine (9), du dichlorvos (organophosphoré), de la crimidine (souricide), de l'embutramide (euthanasiant) (10) et du White spirit $\circledast$ ont été retrouvés chacun dans un cas.

\section{Associations entre les différentes sub- stances}

Le tableau I regroupe les dossiers analysés selon les associations entre les différentes classes de substances. Ainsi parmi les 272 dossiers d'autopsies, 144 dossiers (53\%) contiennent un seul type de substances, pour la plupart des médicaments (69 dossiers). De l'alcool seul a été retrouvé dans 52 dossiers (associé le plus souvent à des noyades, pendaison ou défenestration), des stupé- fiants seuls dans 15 dossiers et du CO dans trois dossiers. Les cinq dossiers classés dans la catégorie « autres $»$ de la figure I contiennent également chacun un seul type de substance.

En ce qui concerne les dossiers pour lesquels seuls des médicaments ont été mis en évidence, il s'agissait la plupart du temps d'intoxications poly-médicamenteuses et notamment avec plusieurs classes de médicaments (benzodiazépines associées à des antidépresseurs ou des neuroleptiques). Les intoxications monomédicamenteuses, notamment par des benzodiazépines, qui sont les médicaments les plus souvent rencontrées, sont peu fréquentes et les décès imputables à ces seules benzodiazépines sont rares (11).

De même, les surdosages par antidépresseurs seuls sont peu fréquents, mais ils sont retrouvés dans des intoxications fatales en association avec d'autres psychotropes (12).

Une étude réalisée en 2001 par Goeringer et coll. montrait que les antidépresseurs (notamment la venlafaxine) étaient la plupart du temps associés à d'autres psychotropes dans les cas de décès (13).

Il est à noter que les substitutifs (méthadone et buprénorphine) ne sont pas retrouvés seuls mais toujours en association, notamment avec des médicaments. Concernant la buprénorphine (14), celle-ci était systématiquement associée à des benzodiazépines.

Ces associations de deux types de substances représentent 103 de nos 272 dossiers $(37,8 \%)$ avec en majorité des associations alcool et médicaments le plus souvent psychotropes, dont l'alcool potentialise les effets pharmacologiques.

Les associations de trois types de substances les plus fréquemment rencontrées sont celles incluant des stupéfiants, des médicaments et de l'alcool ( 6 dossiers sur les 18 de cette catégorie de trois types de substances).

Seuls sept dossiers contenaient des substitutifs, des médicaments, des stupéfiants et de l'alcool.

\section{Relations entre conclusions d'autopsies et résultats toxicologiques (tableau II)}

Une première catégorie regroupe les dossiers pour lesquels la cause du décès est considérée comme toxique a priori. Cette catégorie compte 80 dossiers parmi les 292 dossiers pour lesquels nous avions accès aux rapports d'autopsies. Dans $85 \%$ des cas, la cause toxique a été confirmée par les analyses toxicologiques.

La deuxième catégorie concerne les causes de décès a priori non toxiques (noyade, homicide, pendaison...). Pour $61,8 \%$ de ces dossiers, l'analyse toxicologique n'a pas apporté d'éléments nouveaux, aucune substan- 
Tableau I : Les associations entre les différentes substances.

\begin{tabular}{|c|c|c|}
\hline & NOMBRE DE DOSSIERS & TYPES DE SUBSTANCES (nombre de dossiers) \\
\hline I seul type de substances & 144 & $\begin{array}{l}\text { - médicaments seuls (69) } \\
\text { - alcool seul (52) } \\
\text { - stupéfiants seuls (15) } \\
\text { - strychnine, crimidine, dichlorvos, embutramide, white spirit (5) } \\
\text { - HbCO (3) }\end{array}$ \\
\hline 2 types de substances & 103 & $\begin{array}{l}\text { - médicaments + alcool (58) } \\
\text { - stupéfiants + alcool (19) } \\
\text { - stupéfiants + médicaments (9) } \\
\text { - substitutifs + médicaments (7) } \\
\text { - HbCO + alcool (7) } \\
\text { - HbCO + médicaments (2) } \\
\text { - HbCO + stupéfiants (1) }\end{array}$ \\
\hline 3 types de substances & 18 & $\begin{array}{l}\text { - stupéfiants + médicaments + alcool (6) } \\
\text { - substitutifs + médicaments + alcool (5) } \\
\text { - substitutifs + médicaments + stupéfiants (3) } \\
\text { - HbCO + médicaments + alcool (2) } \\
\text { - substitutifs + stupéfiants + alcool (2) }\end{array}$ \\
\hline 4 types de substances & 7 & - substitutifs + médicaments + stupéfiants + alcool (7) \\
\hline
\end{tabular}

Tableau II : Relations entre conclusions d'autopsies et résultats toxicologiques.

\begin{tabular}{|c|c|c|c|}
\hline $\begin{array}{l}\text { CONCLUSIONS } \\
\text { D'AUTORSIES }\end{array}$ & $\begin{array}{l}\text { NOMBRE DE } \\
\text { DOSSIERS }\end{array}$ & $\begin{array}{l}\text { CONCLUSIONS } \\
\text { TOXICOLOGLUES }\end{array}$ & $\begin{array}{l}\text { NOMABRE DE } \\
\text { BOSSIERS }\end{array}$ \\
\hline \multirow{2}{*}{$\begin{array}{l}\text { Cause du décès connue } \\
\text { (toxique a priori) }\end{array}$} & \multirow{2}{*}{80} & confirmé & 68 \\
\hline & & non confirmé & 12 \\
\hline \multirow{3}{*}{$\begin{array}{l}\text { Cause du décès connue } \\
\text { (non toxique a priori) }\end{array}$} & \multirow{3}{*}{181} & $\begin{array}{l}\text { résultats non toxiques ou absence } \\
\text { de substance retrouvée }\end{array}$ & 112 \\
\hline & & $\begin{array}{l}\text { présence de substances pouvant avoir un rapport } \\
\text { direct ou indirect avec la cause connue du décès }\end{array}$ & 62 \\
\hline & & $\begin{array}{l}\text { résultats toxiques, le décès aurait pu survenir par } \\
\text { la présence de toxiques }\end{array}$ & 7 \\
\hline \multirow{2}{*}{ Mort naturelle? } & \multirow{2}{*}{31} & Mort toxique & 4 \\
\hline & & Mort non toxique & 27 \\
\hline
\end{tabular}

ce n'ayant été retrouvée ou les substances retrouvées ne pouvant être incriminées dans les causes de décès. En revanche, dans $34 \%$ de ces cas a priori non toxiques, les analyses toxicologiques ont permis de mettre en évidence la présence de substance pouvant avoir un rapport direct ou indirect avec la cause connue du décès. Nous citerons, en exemple, un cas de décès par polytraumatisme suite à une chute du sixième étage. L'analyse toxicologique avait mis en évidence une concentration sanguine de $2,34 \mathrm{~g} / \mathrm{l}$ d'alcool, pouvant avoir été une cause de cette chute.
Dans cette même catégorie de décès a priori non toxiques, pour 7 des 181 dossiers analysés $(3,8 \%)$, les résultats toxicologiques ont établi que ces décès auraient pu survenir par la seule présence de toxiques (par exemple, intoxication massive suivie d'une pendaison).

La dernière catégorie rassemble les 31 des 292 dossiers pour lesquels l'autopsie avait pressenti une mort naturelle. $27(87 \%)$ de ces cas ont montré l'absence de tout toxique et $4(13 \%)$ ont révélé un décès pouvant être d'origine toxique. La première associait du diazépam, 
du nordiazépam et de l'oxazépam à des concentrations thérapeutiques et une alcoolémie à $3,25 \mathrm{~g} / \mathrm{l}$. La seconde montrait une prise probable d'héroïne avec morphine à $162 \mathrm{ng} / \mathrm{ml}$, codéine $(10 \mathrm{ng} / \mathrm{ml})$ et alcool à $1,0 \mathrm{~g} / \mathrm{l}$. La troisième associait de la lévomépromazine à très forte concentration $(1040 \mathrm{ng} / \mathrm{ml})$, du zuclopentixol, du diazépam, du nordiazépam et de l'acide valproïque, tous à concentrations thérapeutiques. Enfin la dernière associait de la buprénorphine $(7,4 \mathrm{ng} / \mathrm{ml})$, de la norbuprénorphine $(6,9 \mathrm{ng} / \mathrm{ml})$, du zolpidem, du bromazépam, du dextropropoxyphène à des concentrations thérapeutiques et une alcoolémie à $3,70 \mathrm{~g} / \mathrm{l}$.

\section{Résultats toxicologiques en l'absence d'autopsie (66 cas)}

Pour 25 des 66 dossiers étudiés (38\%), l'analyse toxicologique a montré la présence de substances ayant $\mathrm{pu}$ entraîner le décès. Pour les 41 autres dossiers, seules des substances à concentrations non toxiques ont été mises en évidence.

\section{Conclusion}

Ce bilan de 30 mois d'expertises toxicologiques a permis de recenser les substances les plus couramment retrouvées lors de recherches de causes de décès. Nous avons ainsi mis en évidence que les médicaments sont les plus fréquemment incriminés (utilisés notamment lors de suicide), soit seuls, soit en association avec l'alcool. Et parmi ces médicaments, les benzodiazépines restent les plus souvent rencontrées, reflet probable d'une forte consommation de ces substances en France.

Cette étude montre également l'intérêt de l'analyse toxicologique lors de la recherche de causes de décès puisqu'elle permet d'expliquer directement près de la moitié des décès pour lesquels une autopsie est pratiquée. Comme le souligne O. Suzuki (15), le nombre de toxiques utilisés étant en constante augmentation, le rôle des analyses toxicologiques est de plus en plus important, les résultats d'analyses toxicologiques apportant souvent des éléments supplémentaires à l'enquête judiciaire.

Une étude comme celle-ci permet d'évaluer de façon précise la nature des substances les plus fréquemment rencontrées dans les recherches de causes de décès, qui peut évoluer dans le temps en fonction des modes de prescriptions. Dans cette perspective, nous continuons à recenser au fur et à mesure des dossiers que nous analysons, toutes les données potentiellement utilisables (substances retrouvées, concentrations, relations conclusions d'autopsies-conclusions toxicologiques).

\section{Références}

1. Cirimele V., Lohner S., Ludes B., Kintz P. Décès liés à l'usage de buprénorphine: investigations toxicologiques dans le cadre de cas medico-légaux. Ann. Toxicol. Anal. $2001 ; 13$ (4) : 260-4.

2. Pépin G., Billault F., Gaillard Y. Analyses toxicologiques réalisables après exhumations de corps. A propos de plusieurs cas en médecine légale. Toxicorama $1999 ; 11$ (3) : 198-9.

3. Lahti R.A., Vuori E. Fatal drug poisonings : medico-legal reports and mortality statistics. Forensic Sci. Int. 2003 $136: 35-46$.

4. Pascal P., Moulsma M., Miras A., Gagnieu M.C., Lardet G., Malicier D., Vallon J.J. Bilan de deux ans d'expertise toxicologique réalisée pour l'Institut de Médecine Légale de Lyon. Toxicorama 1996 ; 8 (4) : 27-33.

5. Duverneuil C., Lorin de la Grandmaison G., de Mazancourt P., Alvarez J.C. A high performance liquid chromatography method with photodiode-array UV detection for therapeutic drug monitoring of the non-tricyclic antidepressant drugs. Ther. Drug Monit. 2003 ; 25 (5) : 565-73.

6. Delamoye M., Paraire F., de Mazancourt P., Alvarez J.C A high performance liquid chromatography method with photodiode-array UV detection for therapeutic drug monitoring of 13 ß-blockers and one metabolite. Forensic Sci. Int. $2004 ; 141(1): 23-31$.

7. Tracqui A., Ludes B. Médicaments psychotropes. In Kintz P. : Toxicologie et pharmacologie médicolégales. Ed : Paris : Elvesier Option/Bio, 1998 ; 233-74.

8. Goullé J.P., Mura P., Pépin G., Dumestre-Toulet V., Kintz P. On ne meurt pas du cannabis ... mais il tue! Toxicorama $1999 ; 11$ (3) : 203.

9. Duverneuil C., Lorin de la Grandmaison G., de Mazancourt P., Alvarez J.C. Liquid chromatography/photodiode-array detection for determination of strychnine in blood: a fatal case report. Forensic Sci. Int. $2004 ; 141$ (1) : 17-21.

10. Abe E., Delamoye M., Mathieu B., Durigon M., de Mazancourt P., Advenier C., Alvarez JC. A rapid and sensitive high-performance liquid chromatography method for the determination of embutramide (a Tanax $®$ or T-61 component) in human blood with photodiode-array detection. J. Anal Toxicol. $2004 ; 28$ (2) : 118-21.

11. Nisse P., Deveaux M., Linke J.C, Ghysel M.H., Peucelle D., Mathieu-Nolf M. Intoxications par des benzodiazépines: revue des 157 décès survenus dans le nord de la France au cours des 5 dernières années. Ann. Toxicol. Anal. $2001 ; 13$ (2) : 117.

12. Deveaux M., Ferroul D., Leman C., Tournel G., Hédouin V., Gosset D. Forensic intoxications by new antidepressants: report of 22 cases. Ann. Toxicol. Anal. 2002; 14 (3) : 308.

13. Goeringer K.E., Mc Intyre I.M., Drummer O.H. Postmortem tissue concentrations of venlafaxine. Forensic Sci. Int. $2001 ; 121: 70-5$.

14. Kintz P. Deaths involving buprenorphine: a compendium of French cases. Forensic Sci. Int. $2001 ; 121$ : 65-9.

15. Suzuki O., Seno H., Watanabe-Suzuki K., Ishii A. Situations of poisoning and analytical toxicology in Japan. Forensic Sci. Int. $2000 ; 113: 331-8$. 